Article

\title{
Interactive Field Effect of Atomic Bonding Forces on the Equivalent Elastic Modulus Estimation of Micro-Level Single-Crystal Copper by Utilizing Atomistic-Continuum Finite Element Simulation
}

\author{
Chang-Chun Lee ${ }^{1, * \mathbb{D}}$ and Jing-Yan $\mathrm{He}^{2}$ \\ 1 Department of Power Mechanical Engineering, National Tsing Hua University, No. 101, Section 2, \\ Kuang-Fu Road, Hsinchu 30013, Taiwan \\ 2 Department of Mechanical Engineering, National Chung Hsing University, No. 145 Xingda Rd., South Dist., \\ Taichung 40227, Taiwan; hjy2018@foxmail.com \\ * Correspondence: cclee@pme.nthu.edu.tw; Tel.: +886-3-5162410
}

Academic Editors: Giuseppe Cirillo and Hom Nath Dhakal

Received: 7 October 2020; Accepted: 31 October 2020; Published: 3 November 2020

\begin{abstract}
This study uses the finite element analysis (FEA)-based atomistic-continuum method (ACM) combined with the Morse potential of metals to determine the effects of the elastic modulus $(E)$ of a given example on atomic-level single-crystal copper $(\mathrm{Cu})$. This work aims to overcome the estimated drawback of a molecular dynamic calculation applied to the mechanical response of macro in-plane-sized and atomic-level-thick metal-based surface coatings. The interactive energy of two $\mathrm{Cu}$ atoms within a face-centered metal lattice was described by a mechanical response of spring stiffness. Compared with the theoretical value, the parameters of the Morse potential dominated the predicted accuracy through the FEA-based ACM. Moreover, the analytic results indicated that the effective $E$ of a single-crystal $\mathrm{Cu}$ was significantly sensitive to the given range of the interactive force field among atoms. The reliable elastic moduli of 86.8, 152.6, and 205.2 GPa along the $\mathrm{Cu}(100)$, $\mathrm{Cu}(110)$, and $\mathrm{Cu}(111)$ orientations of the $\mathrm{Cu}$ metal were separately acquired using the presented FEA-based ACM methodology.
\end{abstract}

Keywords: atomistic-continuum method; morse potential; atomic bond; finite element analysis; elastic modulus

\section{Introduction}

With the rapid development and widespread utilization of flexible optoelectronic devices, macro in-plane metal-based surface coatings with an atomic-level thickness and nanowires composed of single-crystal copper $(\mathrm{Cu})$ are being utilized as electrodes because of their outstanding electronic and mechanical responses [1,2]. The mechanical properties of the $\mathrm{Cu}$ metal vary significantly from the macroscale to the nanoscale. In addition, the orientation of crystal lattices is influenced by anisotropy behavior. Consequently, the foregoing mechanical properties of thin films scaled down to the nanoscale or atomic level are difficult to acquire through experiments. In previous work, the elastic moduli of single-crystal $\mathrm{Cu}$ at the lattice orientations of (100), (110), and (111) were reported as 66.7, 130.3, and 191.1 GPa, respectively [3]. The abovementioned values are estimated by elasticity solid mechanics with the integration of measured stiffness and compliance constants. Compared with the analytical solution, smaller values were measured by microcantilever testing for single-crystal $\mathrm{Cu}$ along the abovementioned lattice orientations (i.e., 43-59, 123-145, and 148-181 GPa, respectively) [4]. Wang et al. [5] adopted nanoindentation to measure the directional modulus of thin $\mathrm{Cu}$ coatings. However, compared with the testing data of other research groups, an obvious deviation was noted 
on the (100) orientation because of an elastic modulus (E) in the range of 115-142 GPa. Dub et al. [6] utilized a similar approach and obtained significantly different testing results because magnitudes of 52 and $170 \mathrm{GPa}$ were obtained for the $\mathrm{Cu}(100)$ and $\mathrm{Cu}(111)$ directions, respectively. Thus, numerous tests have been conducted to assess the anisotropic mechanical properties at the nanoscale or atomic level. However, the experimental procedures for atomistic-scale coating, which can induce geometry/size effects, still present serious deviations, even if testing preparations were made. Some mechanical properties lead to the predicted difficulty of device reliability. Thus, simulation has been adopted to obtain the nanoscale mechanical properties of ultrathin metal coatings to eliminate geometric complexity and measured uncertainty. Molecular dynamics (MD) is one of the popular numerical methods for predicting the properties of atomic-scale metals. Ahadi et al. [7] employed MD by applying a finite tensile strain to investigate the size-dependent Poisson's ratio of crystal Cu nanobeams having crystallographic orientations. Moreover, the yield point and directionality of $\mathrm{Cu}$ nanotubes under a three-axial tension can be estimated through MD [8]. Similar numerical procedures were used to extract the temperature-dependent plastic deformation of $\mathrm{Cu}$ nanowires when the related stress-strain curve was completely acquired [9]. The finite element analysis (FEA)-based atomic-continuum method $(\mathrm{ACM})$ is a promising alternative to MD simulation for studying materials emulating mechanical properties, such as carbon nanotubes (CNTs), as the latter require intensive calculations. Li et al. [10] were the first to propose that the interaction between a pair of carbon atoms linked by a chemical bond could be equivalent to a beam with two nodes. Using ACM theory, Tserpes et al. [11] used FEA to calculate the Young's modulus of a single-wall CNT that replaced the covalent bonds between carbon atoms with a beam element having six degrees of freedom. Previous studies on ACM simulation used spring elements to explore the shear modulus and vibration [12-15]. The abovementioned approaches are widely adopted in the investigation of different materials, especially material characteristics highly dependent on the lattice or microstructure. $\mathrm{Cu}$, one of the widely utilized materials in semiconductor processing, also has many characteristics that need to be explored by the utilization of MD and FEA-based ACM approaches. The plasticity and void growth behavior of copper are enabled by atomistic modeling and statistical analysis [16,17]. Moreover, some publications are focused on the estimation of the fracture properties of single crystal $\mathrm{Cu}$ by similar approaches. Lee et al. [18] used the interatomic potential function of the embedded-atom method to model face-centered cubic structures and to analyze the mode-I fracture behavior under various magnitudes of T-stress. The fractal theory is adopted to interpolate the surface roughness and morphology influence between the $\mathrm{Cu}$ rigid plane and elasto-plastic rough substrate [19]. Furthermore, the mixed-mode fracture characteristics of single crystal $\mathrm{Cu}$ are revealed to induce the transition of fracture patterns as the incensement of fracture mode mixity [20]. Compared with general atomistically calculated approaches such as MD, the FEA-based ACM method requires fewer computing resources and satisfies the requirements of effective material properties applied to large simulated models. Consequently, the mechanical properties estimated using FEA-based ACM may be related to the characteristics of thin films constructed in a global device model for reliability predictions and are beneficial for design improvements, such as the flexibility of device applications. As previously mentioned, the modeling scope of the MD simulation, which considers the bonding interactions among the considered atoms, is quite small (less than 10,000) because of hardware limitations, such as central processing unit and memory. Thus, MD calculation is unsuitable for emulating the effects of atomic-level coating on an entire structure within a large-scale estimated model. To resolve this problem, the extraction of the $E$ of atomic-level single-crystal $\mathrm{Cu}$ through FEA-based ACM is proposed. Given that the considered range of the interactive force field among $\mathrm{Cu}$ atoms is extended to a lattice length of $3.61 \AA$, several kinds of spring stiffnesses, adopted in the ACM model, are necessary to describe the effects of the different levels of bonding strength on the atomic-level mechanical characteristics. Thus, the bonding effect among atoms at neighboring levels on the estimated effective $E$ of atomic-scale $\mathrm{Cu}$ metal was discussed in detail. In addition, five sets of Morse potential parameters, used to describe the bonding behavior of $\mathrm{Cu}$ atoms, were examined for their predicted accuracies on the effective modulus. 


\section{Potential Function of Cu Metal Atoms}

\subsection{Morse Potential}

The Morse potential is typically utilized because of its general suitability for metallic bonds and simple formula structure for various fields, such as nanoindentation and machine tool cutting optimization [21-25]. In accordance with Girifalco and Weizer's demonstration for calculating second-order elastic constants [26], the Morse potential is useful to the atomic properties of metals. The interaction energy $V_{i j}$ of a pair of atoms $i$ and $j$ is expressed as:

$$
V_{i j}=D\left\{\exp \left[-2 \alpha\left(r_{i j}-r_{0}\right)\right]-2 \exp \left[-\alpha\left(r_{i j}-r_{0}\right)\right]\right\}
$$

where $r_{i j}$ is the distance between the atoms $i$ and $j$. The symbols $D$ and $\alpha$ represent the depth of the interatomic potential well and the force constant, respectively. The index $r_{0}$ is the equilibrium distance between the atoms $i$ and $j$. The interatomic force $F$ of pairwise atoms can be obtained by derivation with respect to the distance $r_{i j}$. Once again, the second derivative of Equation (1) was implemented to gain the stiffness $k$ of two isolated atoms of $i$ and $j$. In this way, the effective spring constant adopted in ACM to describe the behavior of atoms was found. The formulas for the interatomic force $F$ and the stiffness $k$ are shown in Equations (2) and (3), respectively:

$$
\begin{aligned}
& -F=\frac{\partial V_{i j}}{\partial r_{i j}}=-2 \alpha D\left\{\exp \left[-2 \alpha\left(r_{i j}-r_{0}\right)\right]-\exp \left[-\alpha\left(r_{i j}-r_{0}\right)\right]\right\} \\
& k=\frac{\partial^{2} V_{i j}}{\partial r_{i j}^{2}}=2 \alpha^{2} D\left\{2 \exp \left[-2 \alpha\left(r_{i j}-r_{0}\right)\right]-\exp \left[-\alpha\left(r_{i j}-r_{0}\right)\right]\right\}
\end{aligned}
$$

\subsection{Comparisons of Selected Morse Parameters}

To achieve a good estimated result of the Cu's E by using FEA-based ACM combined with the Morse potential functions, suitable and accurate Morse parameters must be determined. Consequently, five parameter sets of Morse parameters for $\mathrm{Cu}$ metal were obtained from different research groups to determine their similarity with the theoretical value and the measured data. Set 1 was provided by Girifalco et al. [26] and can extract the elastic constants for face-centered and body-centered metals, including $\mathrm{Au}, \mathrm{Ag}$, and $\mathrm{Cu}$. The constants were calculated by using experimental values, such as the vaporization energy, lattice constant, and compression. Set 2 was obtained from Cotterill et al. [27]. The atom arrangement at the dislocated edge was depicted by central-force approximation with a reflected truncation. Various energy functions, including the Morse potential, were used to calculate the stacking-fault energy, which was highly dependent on the potential form and truncated dimension. In set 3, the relevant constants were based on the second-order and third-order elastic constants of cubic metals such as $\mathrm{Cu}, \mathrm{Ag}$, and $\mathrm{Al}$ [28]. The interaction of atoms was described based on the Morse parameters, which were determined based on the physical characteristics of the bulk modulus and cohesive energy. Another set of potential constants was obtained from a nanoscale machining study of tool wear and fraction [29]. In set 4, MD combined with the Morse potential was used to calculate the friction of dissimilar materials at the tool-work interface. Similarly, the Morse parameters of set 5 were subjected to an MD simulation to explore the phonon dispersion curves and the dependence of the Debye temperature for $\mathrm{Cu}$ lattices [30]. The calculated results agreed well with the experimental data. Table 1 lists the Morse parameter sets from these five resources. Moreover, the relations between the applied force and elongation $\left(\delta\right.$, with respect to $\left.r_{0}\right)$ of a pair of $\mathrm{Cu}$ atoms on the basis of the corresponding Morse parameters are shown in Figure 1. 




Figure 1. A force-elongation $(\delta)$ curve of the $\mathrm{Cu}-\mathrm{Cu}$ atomic bond calculated by the Morse parameters of the present five sets $\left(1 \AA=10^{-10} \mathrm{~m}\right)$.

Table 1. A list of five sets of Morse parameters considered for ACM-based FEA.

\begin{tabular}{ccccc}
\hline Parameter Set No. & $\boldsymbol{D}(\mathbf{e V})$ & $\boldsymbol{\alpha}\left(\mathbf{A}^{-\mathbf{1}}\right)$ & $\boldsymbol{r}_{\mathbf{0}}(\mathbf{\AA})$ & Ref. \\
\hline 1 & 0.343 & 1.356 & 2.866 & {$[26]$} \\
2 & 0.324 & 1.294 & 2.913 & {$[27]$} \\
3 & 5.259 & 1.312 & 2.899 & {$[28]$} \\
4 & 0.343 & 1.356 & 2.626 & {$[29]$} \\
5 & 0.162 & 2.093 & 2.616 & {$[30]$} \\
\hline
\end{tabular}

\section{Atomistic-Continuum Method in FEA Modeling}

As mentioned in the previous section, a FEA-based ACM was used in this study. The simulation work is performed by the commercial FEA software ANSYS 18.0. The fundamental concept of ACM is that the atoms and bonding force are replaced by nodes and springs with varying stiffnesses. For single-crystal $\mathrm{Cu}$, a 3D unit cell with a face-centered arrangement of atoms was constructed in order to form a finite element model with the required size scope. A schematic diagram of single crystal $\mathrm{Cu}$ with different levels of the nearest neighbor atoms is illustrated in Figure 2. To complete the atomic-level modeling of single-crystal $\mathrm{Cu}$, the procedure flow is described below. First, the node was considered as atoms and, in order to build the cubic single crystal structure of $\mathrm{Cu}$, its lattice constant was $3.61 \AA$, this distance being defined as the symbol $a$. For single crystal $\mathrm{Cu}$, the four nearest neighbor atoms were totally contained in its cubic structure. The distance between and the first nearest $\left(\lambda_{1}\right)$ neighbor atom was $2.56 \AA(0.71 a)$, and that between any $\mathrm{Cu}$ atom and the second nearest $\left(\lambda_{2}\right)$ neighbor atoms was $3.61 \AA$ (a), which was equal to the lattice constant of $\mathrm{Cu}$ itself. The distances of the third nearest $\left(\lambda_{3}\right)$ and fourth nearest $\left(\lambda_{4}\right)$ neighbor atoms were $4.43(1.22 a)$ and $6.26 \AA(1.73 a)$, respectively. As the interaction of $\mathrm{Cu}$ atoms has a form similar to a force field, the effective range is considered from the first to the fourth nearest neighboring atom and has a cut-off radius of $6.26 \AA$. In the present FEA-based ACM procedure, the spring elements have an equivalent spring constant $k$, which is calculated and utilized to simulate the relationship between loads versus the displacement of atomic bonding between $\mathrm{Cu}$ atoms. The corresponding effective spring constants of four different neighboring levels of $\mathrm{Cu}$ atoms, calculated with the Morse parameters of the five sets, were calculated using Equation (3), the detailed calculation flow being described as follows. First, we adopted the constant parameters $D, \alpha$, and $r_{0}$ into Equation (3). Then, we utilized the considered $r_{i j}$ to calculate the corresponding effective $k$. For example, the $r_{i j}$ was considered as $\lambda_{1}$ for the $k_{1}$ calculation. In a similar way, the $k_{2}, k_{3}$, and $k_{4}$ could be separately estimated. It is worth mentioning that the foregoing $k$ values are in $\mathrm{eV} /\left(\AA^{-1}\right)^{2}$. With




the final estimated $k$ values in the $\mathrm{nN} / \AA \AA$ unit can be obtained and are summarized in Table 2. Following the abovementioned procedure, the atomic-level FEA modeling of single crystal $\mathrm{Cu}$, considering the first to fourth nearest neighbor atoms' bonding and its boundary conditions for the tensile test, is illustrated in Figure 3. Figure 3a represents the modeled atoms (nodes) and interactive bonding between the $\mathrm{Cu}$ atoms of the FEA model. In addition, the boundary condition setting is shown in Figure $3 b$, with a $0.5 \%$ tensile strain being loaded at the top and bottom surface of the $\mathrm{Cu}$ crystal. That is to say, a $0.1 \%$ tensile strain is applied to extract the effective $E$ from a $\mathrm{Cu}$ crystal with different size domains. In this study, the lattice planes of (100), (110), and (111) were chosen as the loaded orientations during the modeling process. After the tensile strain was induced, the reaction forces were captured in order to calculate $E$ in accordance with the generalized Hooke's law.

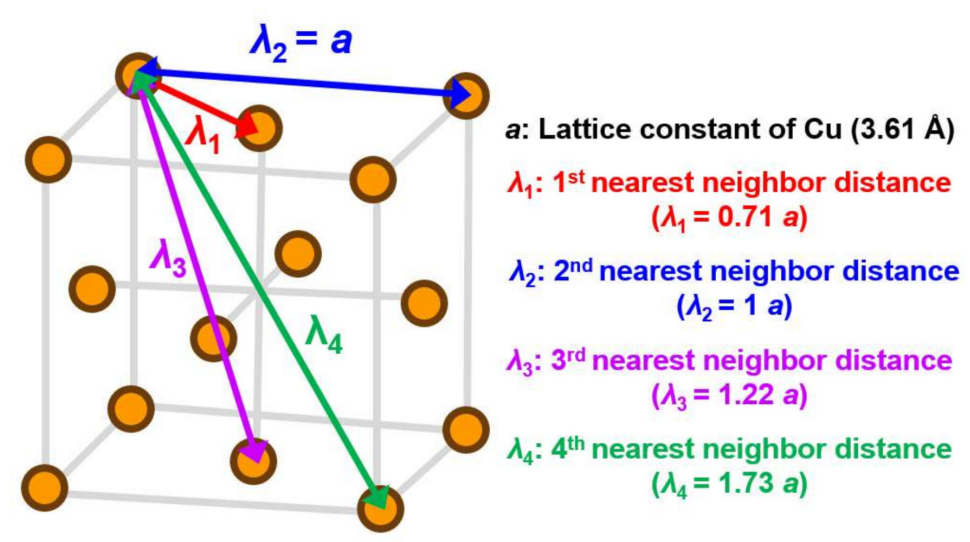

Figure 2. A diagram of the bonding distance from the 1st to the 4th nearest-neighbor distances between atoms $\left(\lambda_{1}, \lambda_{2}, \lambda_{3}\right.$, and $\left.\lambda_{4}\right)$ within a face-centered lattice cell unit.

\section{FEA modeling of $\mathrm{Cu}$ unit cell with $\lambda_{1}$ to $\lambda_{4}$}
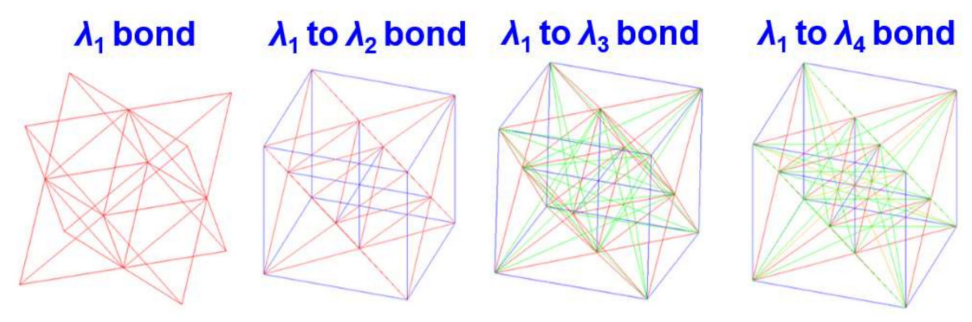

(a)

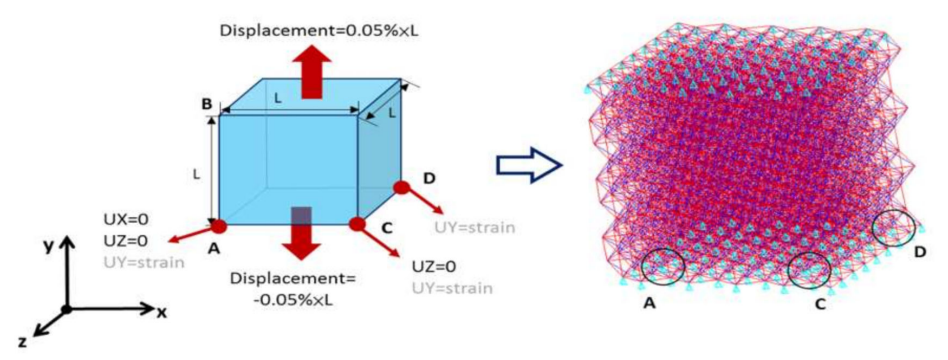

(b)

Figure 3. The atomic-level FEA modeling of single crystal $\mathrm{Cu}$ : (a) Modeled node (atom) and interactive bonding between atoms, considering first to fourth nearest neighbor atoms' bonding; (b) Boundary conditions of single crystal $\mathrm{Cu}$ with a cube having a length of $30 \mathrm{Cu}$ lattice constants (3.61 $\AA$ ) when a $0.1 \%$ tensile uniaxial strain is loaded. 
Table 2. Effective spring constants of the 1st to 4 th nearest neighbor atoms utilized in ACM-based FEA.

\begin{tabular}{|c|c|c|c|c|}
\hline Parameter Sets No. & $\mathrm{k}_{1}(\mathrm{nN} / \mathrm{A})$ & $\mathrm{k}_{2}(\mathrm{nN} / \mathrm{A})$ & $\mathrm{k}_{3}(\mathrm{nN} / \mathrm{A})$ & $\mathrm{k}_{4}(\mathrm{nN} / \mathrm{A})$ \\
\hline 1 & 6.286 & -0.202 & -0.185 & -0.020 \\
\hline 2 & 5.998 & -0.135 & -0.176 & -0.022 \\
\hline 3 & 97.090 & -2.480 & -2.853 & -0.343 \\
\hline 4 & 2.676 & -0.253 & -0.145 & -0.014 \\
\hline 5 & 3.262 & -0.211 & -0.049 & -0.001 \\
\hline
\end{tabular}

\section{Results and Discussion}

\subsection{Estimated Accuracies of the Morse Parameters}

To determine the Morse parameters that can be combined with the present ACM simulation and acquire good estimated results as compared with the theoretical solutions, the estimated results obtained using the parameters provided from the five sets were compared with each other. Given the selected example of the predicted results for the orientation of $\mathrm{Cu}(110)$ (Figure 4), the range impacts of the force field truncated among the $\mathrm{Cu}$ atoms are included by increasing the cut-off levels of the nearest neighboring atom under a fixed cubic volume with an edge length of $30 a(a=3.61 \AA)$. In other words, considering the different spring constants in the ACM model, the interactive forces among the atoms of a $\mathrm{Cu}$ lattice would further approach the bonding conditions of the actual $\mathrm{Cu}$ crystal structures. The analytic results revealed that the estimated result of $154.1 \mathrm{GPa}$, obtained by introducing the Morse parameter of set 5 into the ACM-based FEA, agreed well with the $E$ of $130.3 \mathrm{GPa}$. The $E$ was reduced from 182.7 GPa to 154.1 GPa when the effective spring constants within the ACM model were accumulatively considered from the first to fourth nearest neighboring atom. In addition, the ACM results of the third and fourth nearest neighboring atoms were nearly identical, implying that the cut-off was sufficiently large to calculate the effect of the atomistic force field at that moment. Consequently, the parameters of set 5 were the best among all the sets because the predicted deviations in other sets were quite evident. Based on the analytical results and discussed consequences, the subsequent results of the ACM analysis were obtained by utilizing the Morse parameters of set 5 .

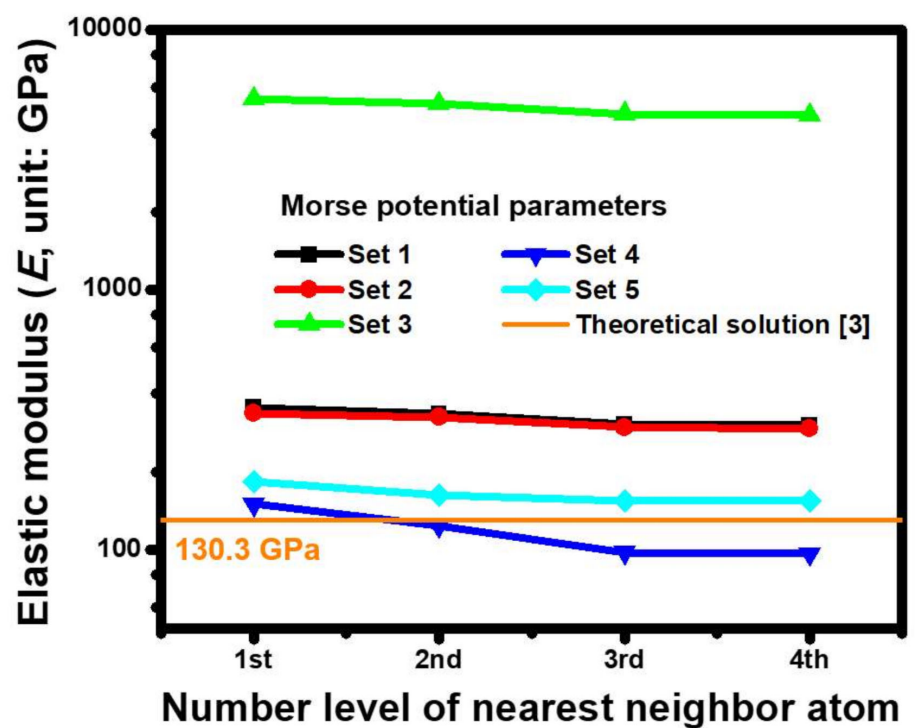

Figure 4. A comparison of $E$ for a $\mathrm{Cu}(110)$ crystal cube with a length of $30 a(a=3.61 \AA)$, estimated by means of the Morse parameters of five sets presented in this study, as various cut-off levels of the pairwise interaction are taken into account. 


\subsection{Oriented Dependence of Atomic-Scale Elastic Modulus (E) Estimated based on ACM Simulations}

Three lattice orientations in a single-crystal $\mathrm{Cu}$ cube, including $\mathrm{Cu}(100), \mathrm{Cu}(110)$, and $\mathrm{Cu}(111)$, were separately built, based on the ACM analysis, in order to extract the corresponding E. As shown by the estimated results of $\mathrm{Cu}(100)$ in Figure 5, the size dependence of the Cu crystal, under various interactive extents between atoms, on the $E$ was analyzed. The $\mathrm{Cu}$ dimensions changed from 10 $a$ to $50 a(a=3.61 \AA)$, which did not significantly influence the mechanical characteristics because the estimated $E$ was only reduced by $3.38 \%$ from $89.71 \mathrm{GPa}$ to $86.77 \mathrm{GPa}$. This phenomenon was attributed to the examined size range from $3.61 \mathrm{~nm}$ to $18.05 \mathrm{~nm}$, which was regarded as being small at the nanoscale level. Consequently, a micro- to macrolevel size effect was excluded in this analysis. Compared to this, the force field effect was significant. When taking the example of a $\mathrm{Cu}$ cube with a $50 a(a=3.61 \AA)$ edge, the effect of the $E$ was only equal to $121.81 \mathrm{GPa}$ if the first nearest bonding force of a pair of atoms was considered. A magnitude of $92.37 \mathrm{GPa}$ was predicted when the second nearest bonding force was included. This behavior was attributed to a fresh force equilibrium within the ACM model of the generated $\mathrm{Cu}$ lattice. When the balanced conditions were further enhanced by considering the other two high-order bonding forces, i.e., the third and fourth nearest interactions of atoms, the foregoing effective $E$ finally converged to $86.8 \mathrm{GPa}$. Similar simulated procedures were adopted, and the estimated ACM results at the orientations of $\mathrm{Cu}(110)$ and $\mathrm{Cu}(111)$ are shown in Figures 6 and 7, respectively. As shown by the converged extent of the calculated modulus in these figures, at least the first three nearest bonding forces should be considered in order to meet the requirements of the range for the interactive force field within a $\mathrm{Cu}$ lattice. Table 3 summarizes the estimated results obtained by ACM analysis proposed in this study and the data collected from previous work. The oriented dependence of the nanoscale effective elastic moduli extracted by ACM simulation was slightly greater than the theoretical values. The reason for such a difference was the size effect. Moreover, the measured data of the $\mathrm{Cu}$ crystals demonstrated that the estimated results were reasonably acceptable because the predicted magnitude was located within the distributed scope of the experimental data.

Table 3. A comparison of crystal-oriented elastic moduli for atomic-scaled $\mathrm{Cu}$ metal extracted from theoretical values, experimental data, and the simulated results of ACM-FEA presented in this study. (Unit: GPa).

\begin{tabular}{cccc}
\hline $\mathbf{C u}(\mathbf{1 0 0 )}$ & $\mathbf{C u ( 1 1 0 )}$ & $\mathbf{C u ( 1 1 1 )}$ & Ref. \\
\hline 66.7 & 130.3 & 191.1 & Theoretical solution [3] \\
$51 \sim 55$ & $121 \sim 134$ & $161 \sim 181$ & {$[4]$} \\
$115 \sim 142$ & $147 \sim 167$ & $140 \sim 169$ & {$[5]$} \\
52 & None & 170 & {$[6]$} \\
86.8 & 152.6 & 205.2 & This study \\
\hline
\end{tabular}

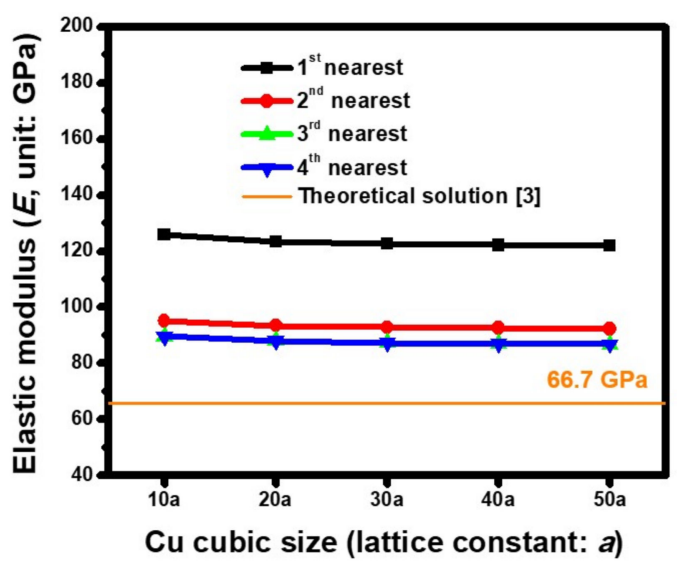

Figure 5. Size-dependent $E$ of the $\mathrm{Cu}(100)$ crystal cube estimated by using the Morse parameters of set 5 when several kinds of cut-offs for interatomic pairwise interaction are considered ( $a=3.61 \AA)$. 


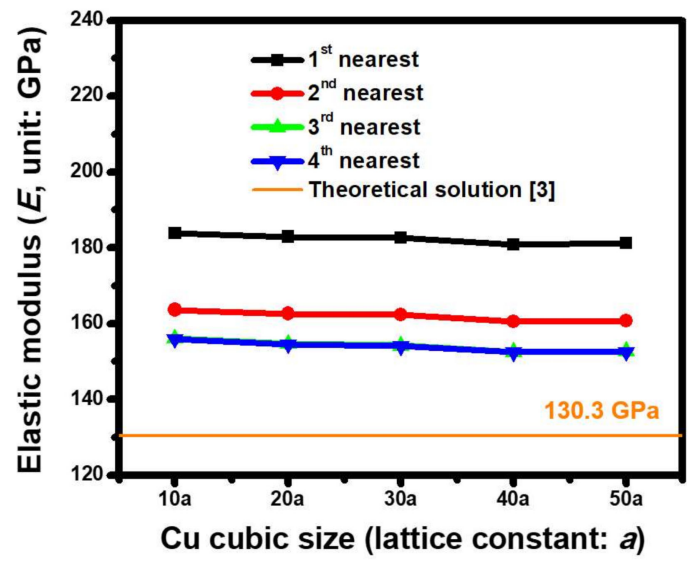

Figure 6. Size dependence of $E$ for the $\mathrm{Cu}(110)$ crystal estimated by utilizing ACM-based FEA when the Morse parameters of set 5 are considered $(a=3.61 \AA)$.

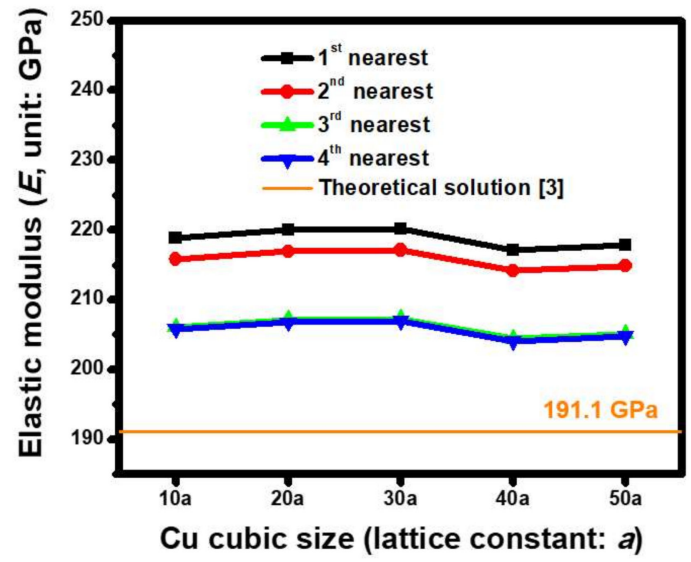

Figure 7. Size dependence of the estimated $E$ acquired by ACM-based FEA combined with the Morse parameters of set 5 when the (111) orientation of the Cu crystal cube is introduced ( $a=3.61 \AA)$.

\section{Conclusions}

For a nanoscale surface coating with a large dimensional area, the corresponding reliability performance under external loadings is difficult to estimate because both the mechanical properties and numerical modeling of the atomic-scale coating must be considered. In this study, the FEA-based ACM was used to resolve this problem through a given example of atomic-level single-crystal $\mathrm{Cu}$. With the assistance of the Morse potential, the interactive behavior among $\mathrm{Cu}$ atoms was successfully described by several kinds of spring stiffnesses within the ACM model. In other words, the $E$ of the nanoscale single-crystal $\mathrm{Cu}$ cube was estimated using FEA by turning the interaction of pairwise atoms into an effective spring constant between two nodes. The analytic results from the ACM simulation indicated that, within an atomic scope, the elastic moduli along the lattice orientations of $\mathrm{Cu}(100), \mathrm{Cu}(110)$, and $\mathrm{Cu}(111)$ were similar to the measured range of the experimental data and the analytical solution. Moreover, the selection of the Morse potential parameter was highly correlated with the estimated mechanical properties when compared with the simulated results when using several sets of parameters. Furthermore, the results revealed that the interactive range of $\mathrm{Cu}$ atoms in the ACM model should take the bonding for the first three nearest neighboring atoms into consideration in order to acquire a more stable $E$ magnitude. 
Author Contributions: Author Contributions: C.-C.L. wrote the presented manuscript, with organizing and designing the analytical procedure of overall study. J.-Y.H. He performed the ACM-based finite element analysis of various copper lattice orientations. All authors have read and agreed to the published version of the manuscript.

Funding: The funding was provided by the Ministry of Science and Technology (MOST), Taiwan, R.O.C., under contract numbers MOST 105-2628-E-007-015-MY3 and MOST 106-2221-E-007-126-MY3.

Acknowledgments: The authors would like to thank the Ministry of Science and Technology (MOST), Taiwan, R.O.C., for providing financial support under contract numbers MOST 105-2628-E-007-015-MY3 and MOST 106-2221-E-007-126-MY3.

Conflicts of Interest: The authors declare that there is no conflict of interests regarding the publication of this paper.

\section{References}

1. Kwon, Y.; Lee, Y.; Kim, S.; Lee, K.; Choa, Y. Full densification of inkjet-printed copper conductive tracks on a flexible substrate utilizing a hydrogen plasma sintering. Appl. Surf. Sci. 2017, 396, 1239-1244. [CrossRef]

2. Liao, Y.; Kao, Z. Direct writing patterns for electroless plated copper thin film on plastic substrates. ACS Appl. Mater. Interfaces 2012, 4, 5109-5113. [CrossRef] [PubMed]

3. Hertzberg, R.W.; Vinci, R.P.; Hertzberg, J.L. Deformation and Fracture Mechanics of Engineering Materials; Wiley: Hoboken, NJ, USA, 2012.

4. Armstrong, D.; Wilkinson, A.; Roberts, S. Measuring anisotropy in Young's modulus of copper using microcantilever testing. J. Mater. Res. 2009, 24, 3268-3276. [CrossRef]

5. Wang, W.; Lu, K. Nanoindentation study on elastic and plastic anisotropies of Cu single crystals. Philos. Mag. 2006, 86, 5309-5320. [CrossRef]

6. Dub, S.; Lim, Y.; Chaudhri, M. Nanohardness of high purity Cu (111) single crystals: The effect of indenter load and prior plastic sample strain. J. Appl. Phys. 2010, 107, 043510. [CrossRef]

7. Ahadi, A.; Melin, S. Size dependence of the Poisson's ratio in single-crystal fcc copper nanobeams. Comput. Mater. Sci. 2016, 111, 322-327. [CrossRef]

8. Yang, Z.; Zhang, G.; Luo, G. Mechanical properties of copper nanocube under three-axial tensile loadings. Chin. Phys. B 2015, 24, 066203. [CrossRef]

9. Wu, H. Molecular dynamics study of the mechanics of metal nanowires at finite temperature. Eur. J. Mech. A-Solids 2006, 25, 370-377. [CrossRef]

10. Li, C.; Chou, T. A structural mechanics approach for the analysis of carbon nanotubes. Int. J. Solids Struct. 2003, 40, 2487-2499. [CrossRef]

11. Tserpes, K.; Papanikos, P. Finite element modeling of single-walled carbon nanotubes. Compos. Pt. B-Eng. 2005, 36, 468-477. [CrossRef]

12. Giannopoulos, G.; Kakavas, P.; Anifantis, N. Evaluation of the effective mechanical properties of single walled carbon nanotubes using a spring based finite element approach. Comput. Mater. Sci. 2008, 41, 561-569. [CrossRef]

13. Fan, F.; Liu, Y.; Hwu, C. Finite element simulation for estimating the mechanical properties of multi-walled carbon nanotubes. Appl. Phys. A-Mater. Sci. Process 2009, 95, 819-831. [CrossRef]

14. Lu, X.; Hu, Z. Mechanical property evaluation of single-walled carbon nanotubes by finite element modeling. Compos. Part B Eng. 2012, 43, 1902-1913. [CrossRef]

15. Sakhaee-Pour, A.; Ahmadian, M.; Vafai, A. Vibrational analysis of single-walled carbon nanotubes using beam element. Thin Walled Struct. 2009, 47, 646-652. [CrossRef]

16. Chandra, S.; Samal, M.K.; Chavan, V.M.; Raghunathan, S. Hierarchical multiscale modeling of plasticity in copper: From single crystals to polycrystalline aggregates. Int. J. Plast. 2018, 101, 188-212. [CrossRef]

17. Chandra, S.; Samal, M.K.; Chavan, V.M.; Raghunathan, S. Void growth in single crystal Copper-an atomistic modeling and statistical analysis study. Philos. Mag. 2018, 98, 577-604. [CrossRef]

18. Lee, G.H.; Chung, Y.J.; Na, S.M.; Beom, H.G. Atomistic investigation of the T-stress effect on fracture toughness of copper and aluminum single crystals. J. Mech. Sci. Technol. 2018, 32, 3765-3774. [CrossRef]

19. Dai, Y.; Lin, J.; Huang, J. Fractal contact behavior of single crystal copper substrate and rigid plane. J. Dispers. Sci. Technol. 2019, 40, 1504-1512. [CrossRef]

20. Cui, C.B.; Lee, G.H.; Beom, H.G. Mixed-mode fracture toughness evaluation of a copper single crystal using atomistic simulations. Comput. Mater. Sci. 2017, 136, 216-222. [CrossRef] 
21. Lin, Y.; Shiu, Y. Effect of crystallographic orientation on single crystal copper nanogrooving behaviors by MD method. Int. J. Adv. Manuf. Technol. 2017, 89, 3207-3215. [CrossRef]

22. Pei, Q.; Lu, C.; Lee, H. Large scale molecular dynamics study of nanometric machining of copper. Comput. Mater. Sci. 2007, 41, 177-185. [CrossRef]

23. Fang, T.; Weng, C. Three-dimensional molecular dynamics analysis of processing using a pin tool on the atomic scale. Nanotechnology 2000, 11, 148-153. [CrossRef]

24. Hsu, Q.; Wu, C.; Fang, T. Deformation mechanism and punch taper effects on nanoimprint process by molecular dynamics. Jpn. J. Appl. Phys. 2004, 43, 7665-7669. [CrossRef]

25. Fang, T.; Jian, S.; Chuu, D. Molecular dynamics analysis of effects of velocity and loading on the nanoindentation. Jpn. J. Appl. Phys. 2002, 41, L1328-L1331. [CrossRef]

26. Girifalco, L.; Weizer, V. Application of the Morse Potential function to cubic metals. Phys. Rev. 1959, 114, 687-690. [CrossRef]

27. Cotterill, R.; Doyama, M. Energy and atomic configuration of complete and dissociated dislocations. I. Edge Dislocation in an fcc Metal. Phys. Rev. 1966, 145, 465-478. [CrossRef]

28. Lincoln, R.; Koliwad, K.; Ghate, P. Morse-potential evaluation of second and third-order elastic constants of some cubic metals. Phys. Rev. 1967, 157, 463-466. [CrossRef]

29. Maekawa, K.; Itoh, A. Friction and tool wear in nano-scale machining- a molecular dynamics approach. Wear 1995, 188, 115-122. [CrossRef]

30. Wette, F.D.; Cotterill, R.; Doyama, M. Lattice dynamics of copper with a Morse potential. Phys. Lett. 1966, 23, 309-311. [CrossRef]

Sample Availability: The material properties regarding the samples of the single-crystal copper are surveyed and available from the authors.

Publisher's Note: MDPI stays neutral with regard to jurisdictional claims in published maps and institutional affiliations.

(C) 2020 by the authors. Licensee MDPI, Basel, Switzerland. This article is an open access article distributed under the terms and conditions of the Creative Commons Attribution (CC BY) license (http://creativecommons.org/licenses/by/4.0/). 